Case Report:

\title{
Penetrating Orbitocranial Injury With a Good Aesthetic and Functional Outcome: A Case Report
}

\author{
Youssef Fahde $^{1^{*}}$ (D), Davis Mpando ${ }^{1}$, Mehdi Laghmari ${ }^{1}$, Houssine Ghannane ${ }^{1}$, Said Aitbenali ${ }^{1}$
}

1. Department of Neurosurgery, Mohammed VI Teaching Hospital, Cadi Ayyad University, Marrakech, Morocco.

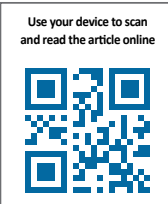

Citation: Fahde Y, Mpando D, Laghmari M, Ghannane H, Aitbenali S. Penetrating Orbitocranial Injury With a Good Aesthetic and Functional Outcome: A Case Report. Iran J Neurosurg. 2021; 7(1):61-66. http://dx.doi.org/10.32598/irjns.7.1.8

doi http://dx.doi.org/10.32598/irjns.7.1.8

\section{(c) (1)}

\section{Article info:}

Received: 13 Jun 2020

Accepted: 11 Nov 2020

Available Online: 01 Jan 2021

Keywords:

Craniofacial Penetrating, Foreign body, Computed tomography, Extraction, Infection

\section{ABSTRACT}

Background and Importance: Transorbitocranial assaults with sharp objects like a knife are rare neuro-ophthalmologic emergencies. However, they can have dramatic functional and life-threatening consequences. Our presentation aims to report the importance of an urgent multidisciplinary approach and to raise awareness among the general population on the importance of preventing violent behavior.

Case Presentation: A 33-year-old man was a victim of a knife attack without obvious brain or ophthalmological lesions. The knife entered the medial part of the orbit. Neurological examination was normal, and Computed Tomography (CT) scan showed intracranial trajectory through the orbit to the frontal horn of the lateral ventricle. The knife was extracted without complications. The patient reported spectacular improvement in visual acuity without neurological or oculomotor deficit at long-term follow-up. In this case report, we will discuss the radiological diagnosis and surgical management of transorbital and orbitocranial injuries by foreign body penetration.

Conclusion: Urgent multidisciplinary management in orbitocranial trauma by stabbing is mandatory to avoid life-threatening complications and irreversible damages.

* Corresponding Author:

Youssef Fahde, MD.

Address: Department of Neurosurgery, Mohammed VI Teaching Hospital, Cadi Ayyad University, Marrakech, Morocco

Tel: +21 (26) 63007507

E-mail: u.fahde@gmail.com 


\section{Highlights}

- Public awareness of the importance of preventing violent behavior should be raised.

- Extraction can prevent worsening damage to vital structures.

- Reasonable radiological interpretation allows choosing a safe surgical procedure.

\section{Plain Language Summary}

Stabbing orbitocranial trauma has serious consequences for the patient's vision, brain, and life. It is a surgical emergency. Our case was admitted for neuro-ophthalmological emergencies. After careful physical examination of the patient, the physician recommended blood tests and $\mathrm{x}$-ray to assess the condition of the eye, bone, brain, and the relationship of the foreign body to the anatomical structures. Magnetic resonance imaging is contraindicated in this type of trauma. Treatment consisted of the extraction of the foreign body without adding any other lesions. The results depend on the severity of the lesions and the patient's clinical condition. To prevent this type of accident, violence must be prohibited by reducing the availability and harmful alcohol consumption and reduce access to bladed weapons.

\section{Background and Importance}

rbitocranial injuries are rare and generally

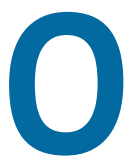

presented with severe brain damage [1].

This type of brain injury represents $0.04 \%$ of all traumatic brain injuries-24\% of open head injury in adults [2]. A multitude of foreign bodies can injure the brain by passing through the orbit. Depending on the direction of the trauma, a foreign body can reach the brain either through the orbital roof, the superior orbital fissure, or the optic canal [3]. The diagnosis is confirmed in most cases when the foreign body is found in the wound or by $x$-ray imaging [4-8]. In this case report of a penetrating brain injury by a knife through the medial aspect of the orbit, we reviewed the radiological findings and surgical management.

\section{Case Presentation}

A 33-year-old man was admitted to the Emergency Department because a knife got stuck in the medial region of his right eye (Figure 1). He felt severe pain in the eye, which became excruciating when attempting to move the eye.

On examination, the knife appeared to be embedded in the globe, with slight bleeding. He had an ecchymosis of the eyelids with a red eye and edematous conjunctiva. The left eye and neurological examination were normal. The globe was intact, the pupil nonreactive with some subconjunctival hemorrhage. Laboratory examinations were normal. He was given intravenous antibiotics and a tetanus booster. The eye was bandaged with gauze, and the patient was transferred to perform a radiological examination Plain skull radiographs confirmed the intracranial place of the knife through the orbital roof (Figure 2).

Computed tomography of the head and face without administration of the contrast material was recommended, and the result showed a $7-\mathrm{cm}$ knife inside the right orbit near the medial canthus, transversing the orbital plate of the frontal bone passing through the right frontal lobe near the frontal horn of ipsilateral ventricle without intraparenchymal hemorrhage or intraventricular inundation (Figure 3).

Cerebral angiography was not performed since it is not available in our department. Moreover, we did not perform it unless the foreign body passes near a sizeable arterial trunk such as the sellar region, Sylvian fissure, or interhemispheric area $[5,6]$. We preferred to manage the closure of the dura defect conservatively because of the damage caused by the approach [5].

In our case, the wound was linear far from LCR lacs and could be patched by the gravitational effect of the brain and arachnoid on the one side and the periorbital and orbital fat on the other side. We brought the patient into the operating room, where the trachea was intubated after general anesthesia. After preparing a pterional approach, we manually extracted the knife taking care not to injure further the contents of the orbit (Figure 4). After several minutes without evidence of external or intracranial bleeding or any change in the patient's vital signs, we concluded the extraction was completed without complication. 


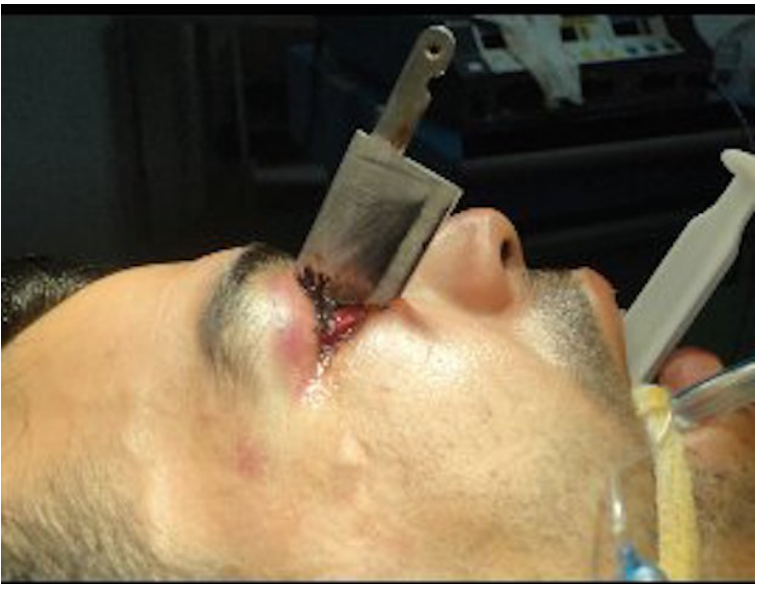

Figure 1. The knife and the entry point in the right eye

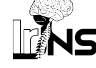

Exploration after extraction found scleral and a lower eyelid wound which an ophthalmologist sutured. Follow-up CT demonstrated no intracranial hemorrhage.

The prognosis was excellent by a spectacular improvement in visual acuity and no neurological or oculomotor deficit. The patient was given antibiotics and eye drops. The long-term outcome was without neuro-ophthalmic sequelae with a very satisfactory aesthetic result (Figure 5).

\section{Discussion}

Penetrating orbitocranial injuries are rare, but they have significant morbidity and mortality (up to $30 \%$ ) [1, 4].

Transorbitocranial stab wounds have a higher incidence of vascular and brain injuries and severe infectious complications [3-9]. The most frequent penetration site is through the orbital plate frontal bone, which

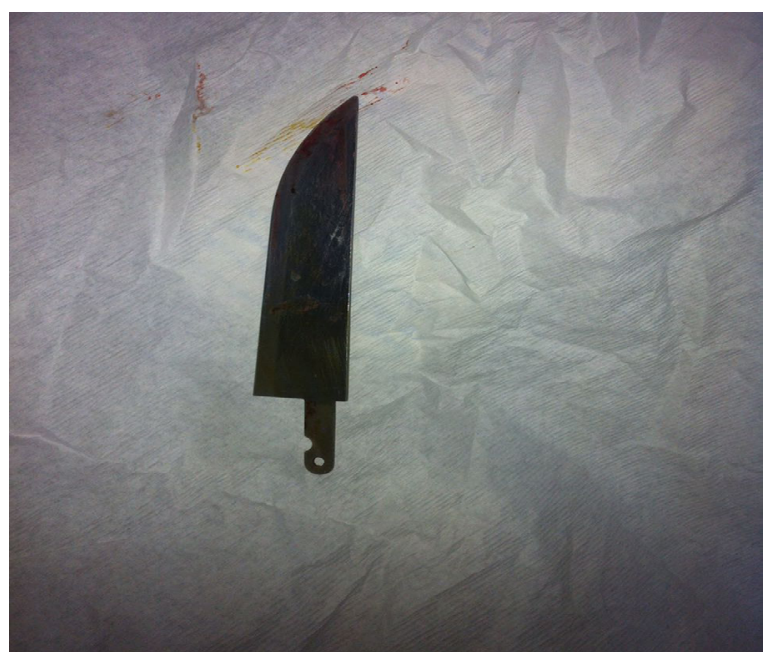

Figure 3. Knife after extraction

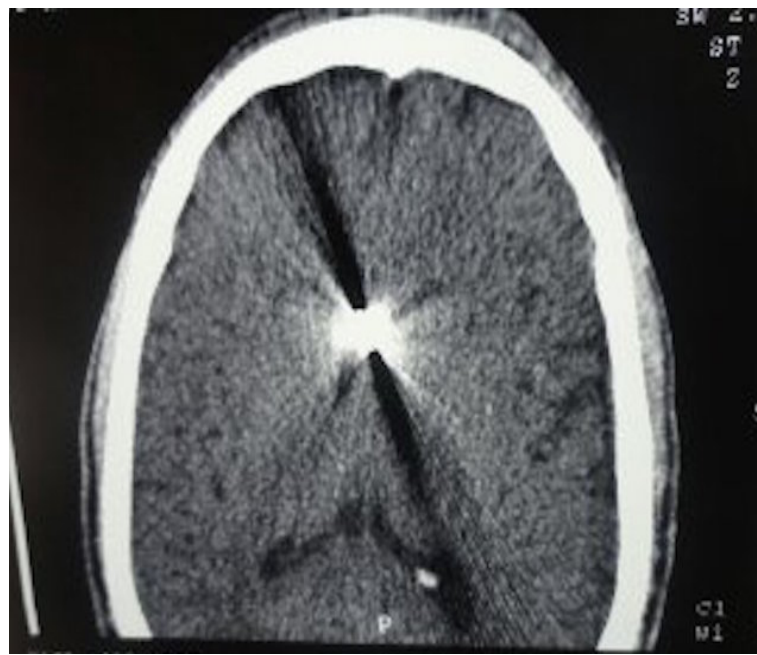

Figure 2. A craniofacial radiography showing the path of the knife from the anterior orbit to the anterior fossa passing through the anterior skull base

is highly vulnerable due to its low thickness. This condition explains the high frequency of frontal lobe contusions, which its prognosis is relatively good [3].

Orbitocranial injuries are explored by craniofacial CT scan. It allows pinpointing lesions caused by penetrating trauma. CT scan determines location and direction of foreign bodies, especially if there is any associated lesion such as calvarial fracture with bone fragments and/or hematoma. It can also help planning surgery to avoid complications. The severity of lesions should be determined as soon as possible as it can identify the lesion extension. Any retained foreign body, location, and inward driven bone fragments that determine the penetrating track, hematoma, and other associated lesions provide helpful information for the planning of the surgical procedure [7, 9].

Immediate complications include globe rupture, retinal detachment, extraocular muscle injury, or optic nerve avulsion. Other complications must be searched as intraventricular pneumocephalus, intraventricular hemorrhage, vascular injury, or brain stem contusion [4].

After eliminating a vascular compromise and foreign bodies far from vital structures, we can remove the foreign body without dissection.

To obtain a good outcome, it is essential to perform an urgent $\mathrm{CT}$ scan to guide an immediate surgery, determine all the lesions for planning extraction of foreign bodies and intervene for any associated urgent situation that can cause the patient's death. Post-operative an- 


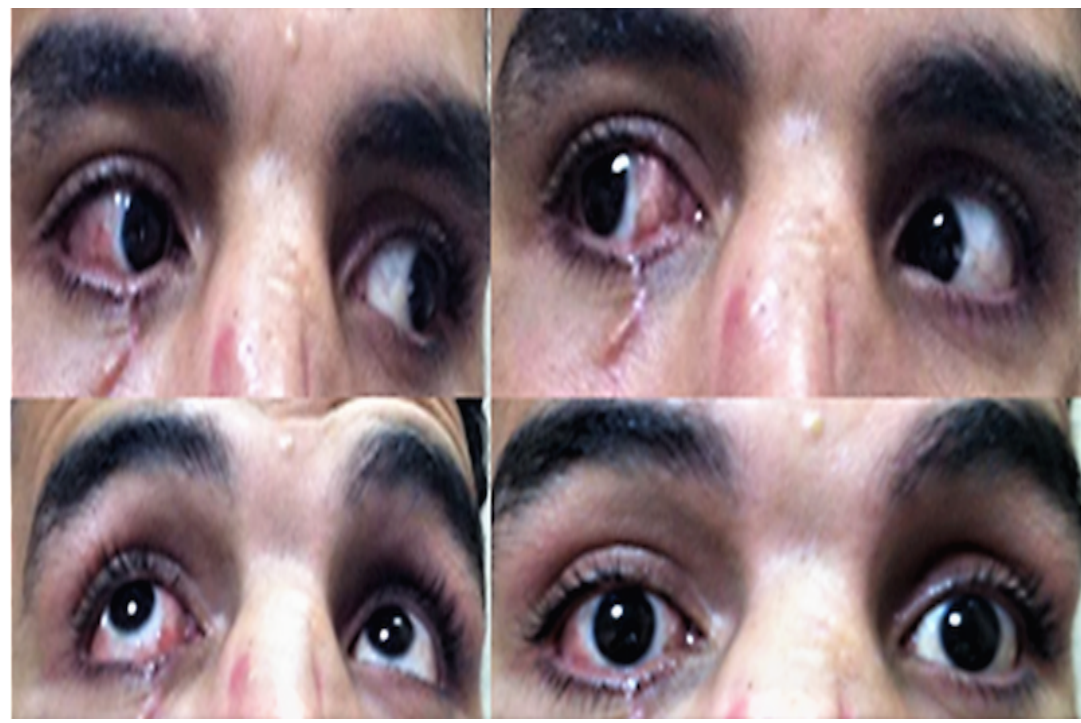

Figure 4. Functional result after 6 months with total preservation of ocular mobility and visual acuity

tibiotics and intensive care avoid immediate complications and help with prognosis [4-9]

\section{Conclusion}

Penetrating orbitocranial trauma by a sharp object is rare. Several lesions can affect the functionality and prognosis. The diagnosis is based on clinical examination and radiological imaging. Urgent multidisciplinary management is mandatory for an excellent therapeutic evaluation. Intracerebral hemorrhage remains a serious complication that can result in life-threatening or significant morbidity.
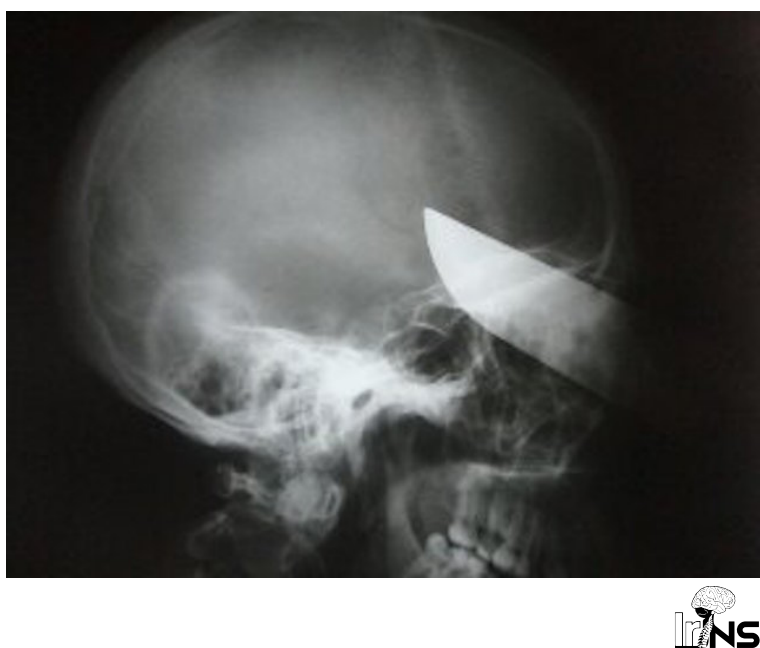

Figure 5. CT scan showing the extent of the knife tip coming into contact with the frontal horn of the right lateral ventricle

\section{Ethical Considerations}

Compliance with ethical guidelines

All ethical principles are considered in this article. The participant was informed about the purpose of the research and its implementation stages. Written informed consent was obtained from the patient.

Funding

This research did not receive any grant from funding agencies in the public, commercial, or non-profit sectors.

\section{Authors' contributions}

Conception and design: Youssef Fahde; Data Collection: Youssef Fahde; Data Analysis and Interpretation: Youssef Fahde, Davis Mpando; Drafting the article: Youssef Fahde, Davis Mpando; Critically revising the article: Mehdi Laghmari, Houssine Ghannane, Said Ait Benali; Reviewing submitted version of manuscript and approving the final version of the manuscript: All authors.

\section{Conflict of interest}

The authors declared no conflict of interest. 


\section{References}

[1] Lin HL, Lee HC, Cho DY. Management of transorbital brain injury. Journal of the Chinese Medical Association. 2007 70(1):36-8. [DOI:10.1016/S1726-4901(09)70299-0][PMID]

[2] Dunya IM, Rubin PA, Shore JW. Penetrating orbital trauma. International Ophthalmology Clinics. 1995; 35(1):25-36. [DOI:10.1097/00004397-199503510-00004] [PMID]

[3] Kitakami A, Kirikae M, Kuroda K, Ogawa A. Transorbitaltranspetrosal penetrating cerebellar injury: Case report. Neurologia Medico-Chirurgica. 1999; 39(2):150-2. [DOI:10.2176/ nmc.39.150] [PMID]

[4] Seçer M, Ergüngör MF, Dalgıç A, Okay HÖ, Dağlıŏglu E Acar OA. Transorbital brain injury by a metalic fragment: A case report. Turkish Neurosurgery. 2007; 17(2):163-5. [PMID]

[5] KazimSF, Shamim MS, Tahir MZ, EnamSA, WaheedS. Management of penetrating brain injury. Journal of Emergencies, Trauma, and Shock. 2011; 4(3):395-402. [DOI:10.4103/09742700.83871] [PMID] [PMCID]

[6] Temple N, Donald C, Skora A, Reed W. Neuroimaging in adult penetrating brain injury: A guide for radiographers. Journal of Medical Radiation Sciences. 2015; 62(2):122-31. [DOI:10.1002/jmrs.101] [PMID] [PMCID]

[7] Agraval A, Pratap A, Agraval CS, Kumar A, Rupakheti S, Transorbital orbitocranial penetrating injury due to bicycle brake handle in a child. Pediatric Neurosurgery. 2007; 43(6):498-500. [DOI:10.1159/000108794] [PMID]

[8] Yamashita T, Mikami T, Baba T, Minamida Y, Sugino T, Koyanagi I, et al. Transorbital intracranial penetrating injury from impaling on an earpick. Journal of Neuro-Ophthalmology. 2007; 27(1):48-9. [DOI:10.1097/WNO.0b013e3180325ef4] [PMID]

[9] Kieck CF, Villiers JC. Vascular lesions due to transcranial stab wounds. J Neurosurg. 1984; 60:42-6. [DOI:10.3171/ jns.1984.60.1.0042] [PMID] 
This Page Intentionally Left Blank 